\title{
Toxoplasma gondii: A mathematical model of its transfer between cats and the environment
}

\author{
Emily K Kelting 1 \\ Advisors: Dr. Brittany E Bannish ${ }^{2}$ and Dr. Sean M Laverty ${ }^{3}$ \\ Department of Mathematics and Statistics \\ University of Central Oklahoma \\ Edmond, OK 73034
}

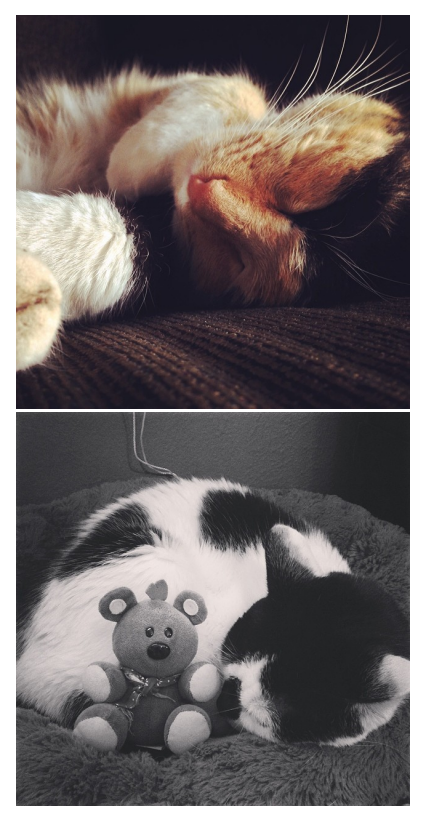

\begin{abstract}
We discuss the transmission of Toxoplasma gondii and its effects in a cat population. Due to the severity of T. gondii spillover infections in pregnant women and monk seals, understanding its dynamics in cats is key to unlocking preventative measures against this parasite. Taking into account susceptible, acutely infectious, and chronically infectious cats, and the surrounding environment, we build a differential equations model of T. gondii transmission in cats. We present our model and the results, identifying conditions under which infection persists. Specifically, we find that higher rates of infection and larger shedding rates facilitate endemic infection. We conclude by discussing how this information can be used to minimize risks to other species.
\end{abstract}

Keywords: Toxoplasma gondii, T. gondii, parasite, cats, environment, mathematical model, differential equations

\section{Introduction}

Discovered in 1908, Toxoplasma gondii is one of the most successful parasites on Earth. In fact, studies show that over half of the world's human population is infected [11. Moreover, this single-celled organism has one of the highest host ranges of any parasite, including cats, humans, rats, and seals [3].

Infection by $T$. gondii comes from consuming infected food, having contact with infected cat feces, or vertical transmission (transfer from mother to fetus) [24]. Although many hosts remain asymptomatic, there are some who suffer clinical toxoplasmosis, the disease caused by the parasite. In humans, symptoms of toxoplasmosis are usually flu-like and include fever, fatigue, body aches, and headaches [25]. Severe reactions can cause pneumonia, hepatitis, and disorders of the eye and central nervous system [14]. Pregnant women and their fetuses most often develop toxoplasmosis, as do non-human mammals such as monk seals [16].

Nearly 200,000 human cases of congenital toxoplasmosis occur each year [26]. Infection

\footnotetext{
1 ekelting@uco.edu

2 bbannish@uco.edu

$3 \overline{\text { slaverty@uco.edu }}$
} 
during pregnancy can result in miscarriages, stillbirths, or birth anomalies [12]. According to one study of 162 T. gondii-infected children, 66 were born "seriously sick," 50 were born with optical impairment, and 38 of those 50 also had a neurological impairment [2]. Another study showed that almost $85 \%$ of women in the United States have little to no immunity against T. gondii during childbearing years. Consequently, these women are highly susceptible to infection [18].

The only animal to which toxoplasmosis is fatal is the monk seal [22]. These toxoplasmosiscaused deaths are detrimental because monk seals are one of the most endangered marine animals in the world [10]. Due to the hardiness of its oocysts (parasite eggs), T. gondii can survive in bodies of water for extended periods of time [24]. This heightens the risk of infection in marine wildlife. One T. gondii-infected Hawaiian Monk Seal that acquired toxoplasmosis suffered from hemorrhaging and presented with prominent lesions on the spleen, brain, and other tissues [16.

Understanding the transmission of T. gondii in cats is crucial, so that strategies can be developed to limit and understand the spillover of $T$. gondii into other species such as the endangered monk seal. It is important to note that cats are the only 'definitive host' of $T$. gondii, meaning they are the only animal in which the parasite reaches maturity and reproduces [14]. The oocysts are then shed into the environment through cat fecal excretions. Because of this, cats are a key part of the parasite's life cycle, so it is vital to study a betweenhost model to show how the parasite passes from cat to cat and potentially spills into other animals. In this paper, we build and analyze a between-host differential equations model to help understand T. gondii transmission via the environment. We specifically study how the shedding rate of parasite into the environment and the subsequent rate of infection affect the proliferation of infection in the cat population. With the results of this investigation, we seek to suggest strategies to pursue (such as treating soil with an agent that kills T. gondii oocysts) to help build a safer environment.

\section{Methods}

Based on the relevant literature and available data on between-host dynamics, we use differential equations to describe the change in $T$. gondii over time and its distribution between hosts. Reinfection is uncommon [1] and only a single genetic strain of the parasite is considered in our between-host model. Furthermore, since stray cats are more prone to infection, we model a feral, rather than domestic, population of cats [4].

We use analytical and computational techniques from differential equations (steady-state and stability analyses, along with numerical solutions) to understand the dynamics of $T$. gondii transmission between cats. From these results, we propose precautions for protecting pregnant women and monk seals from spillover of the parasite from the cat population.

\subsection{Conceptual Model}

The model considers state variables representing the numbers of individuals in three distinct cat subpopulations - susceptible $(S)$, acutely infectious $(I)$, and chronically infectious $(C)$ - 
as well as the number of oocysts in the surrounding environment $(E)$. Cats move between the different subpopulations and transfer $T$. gondii to the environment as depicted in Figure 1 .

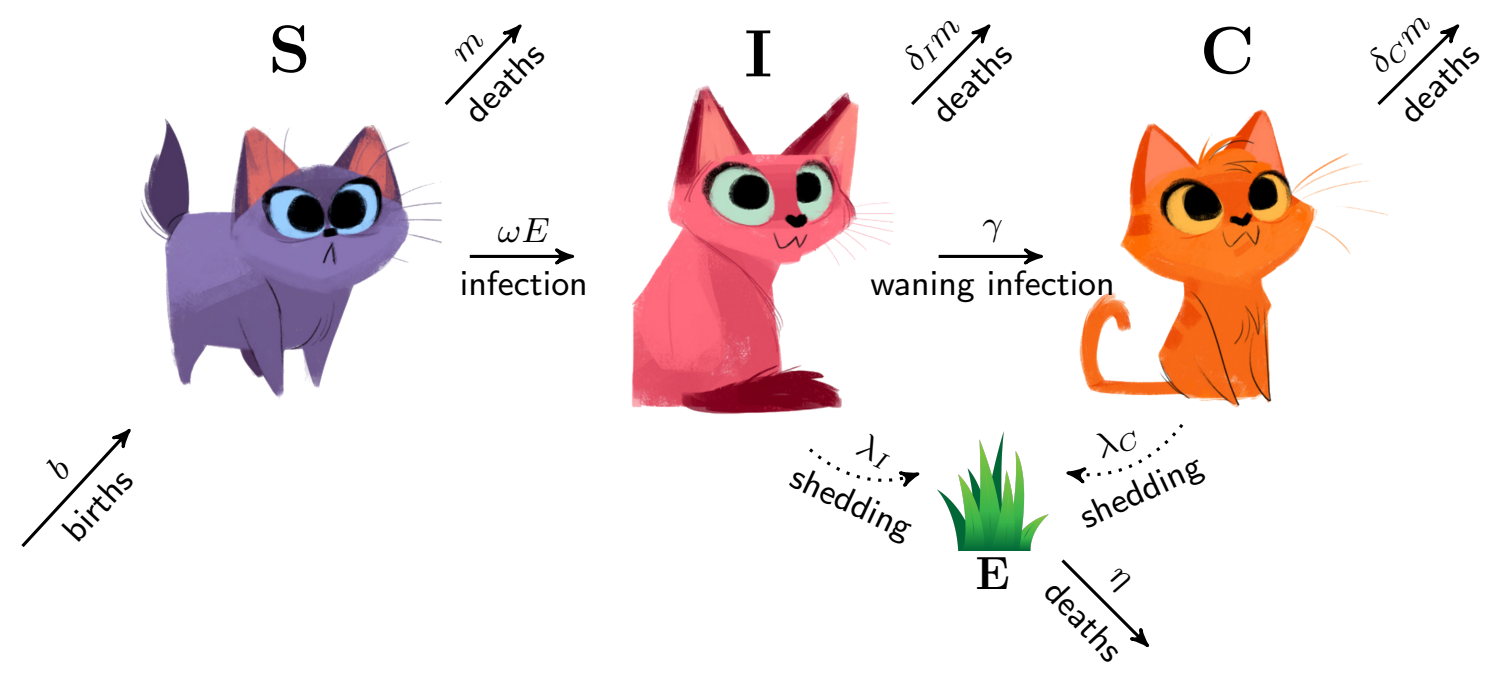

Figure 1: Conceptual model of the transfer of T. gondii between cats and the environment. $S$ : number of susceptible individuals; $I$ : number of acutely infectious individuals; $C$ : number of chronically infectious individuals; $E$ : number of oocysts in the environment. (Image credits: modified from the work of H. Neisham from Daily Cat Drawings).

For simplicity, we assume a constant birth rate of susceptible cats independent of the total population. Furthermore, we assume that all cats are born susceptible due to the low likelihood of vertical transmission of T. gondii in cats [1]. The number of susceptible cats decreases due to infection and mortality. Acute infections occur when susceptible cats acquire the parasite from a contaminated environment. These acutely infectious cats are lost to mortality and to the onset of chronic infection. Loss of acute infection leads to an increase in the chronically infectious cat subpopulation. Once a cat is infected, it remains infected for life. Hence, the only losses to the chronically infectious subpopulation occur by mortality. The environment gains the parasite primarily from acutely infectious cats shedding the oocysts through excrement, but chronically infectious cats can shed at a lower rate [24, 19]. We assume that the oocyst shedding rate by chronically infectious cats is zero, but for generality we show the term in the model. Currently, the only way for $T$. gondii to leave the environment is for the oocysts to degrade.

In 2012, Feng et al. proposed a similar model of the between-host dynamics of T. gondii in a cat population. While their model also focuses on the environment, it does not consider chronic infection. We find this to be a vital component of this model. Without chronically infected individuals, acutely infectious cats will keep shedding oocysts. This leads to an over-saturated environment since there is no reduction in parasite shedding that comes with the chronic stage [9].

Another study that models between-host dynamics is González et al. This paper considers the interaction of cats and humans rather than cats and the environment. Concentrating on 
the cat dynamics, one can find the similarities and differences between our models. Much like our model, cats cannot recover from T. gondii infection. However, the González et al. model allows for the probability of vertical transmission in cats and identifies it as a key mechanism for the transmission of T. gondii in cats [15]. Since the probability of vertical transmission in cats is very low, we neglected it in our model. To the best of our knowledge, our model is the first to study indirect $T$. gondii transmission via the environment in cats with a chronically infectious subpopulation.

\subsection{Model Equations and Parameters}

Based on the dynamics described in Section 2.1, we build the differential equations model (Equations (1)-(4)). The relationships between variables are illustrated in Figure 1 and the parameters are summarized in Table 1.

$$
\begin{aligned}
\text { Susceptible Cats } & \frac{d S}{d t}=b-\omega E S-m S \\
\text { Acutely Infectious Cats } & \frac{d I}{d t}=\omega E S-\gamma I-\delta_{I} m I \\
\text { Chronically Infectious Cats } & \frac{d C}{d t}=\gamma I-\delta_{C} m C \\
\text { Oocysts in Environment } & \frac{d E}{d t}=\lambda_{I} I+\lambda_{C} C-\eta E
\end{aligned}
$$

Table 1: Model parameters. When necessary, subscript $j$ takes values of $C$ or $I$

\begin{tabular}{|c|c|c|c|c|}
\hline Symbol & Meaning & Range of Values & Units & Reference \\
\hline$b$ & $\begin{array}{l}\text { reproduction or birth rate of } \\
\text { cats }\end{array}$ & $\frac{30}{52}$ to $\frac{90}{52}$ & $\frac{\# \text { cats }}{\text { wk }}$ & 20 \\
\hline$m$ & mortality or death rate in cats & $\frac{1}{520}$ to $\frac{3}{520}$ & $\frac{1}{\mathrm{wk}}$ & [13] \\
\hline$\gamma$ & loss of acute infection in cats & $\frac{7}{15}$ to 1 & $\frac{1}{\mathrm{wk}}$ & [1] \\
\hline$\delta_{j}$ & $\begin{array}{l}\text { infection associated mortality } \\
\text { in cats }\end{array}$ & 1.0005 to 1.05 & - & - \\
\hline$\eta$ & $\begin{array}{l}\text { oocyst degradation rate in the } \\
\text { environment }\end{array}$ & $\frac{3}{52}$ to $\frac{5}{52}$ & $\frac{1}{\mathrm{wk}}$ & 24 \\
\hline$\lambda_{j}$ & oocyst shedding rate per cat & $\frac{7}{11} \times 10^{6}$ to $\frac{7}{11} \times 10^{8}$ & $\frac{\# \text { ooc }}{\mathrm{cat} \cdot \mathrm{wk}}$ & [1] \\
\hline$\omega$ & rate of infection in cats & 0.01 to 0.15 & $\frac{1}{\mathrm{wk} \cdot \# \mathrm{ooc}}$ & - \\
\hline
\end{tabular}
to represent chronically or acutely infectious cats, respectively. As a baseline parameter set, we take the midpoint of each parameter range. See Appendix for discussion of parameter values. 
Note that acute infection of $T$. gondii results in the shedding of $10^{6}-10^{8}$ oocysts for 7-15 days after infection [1]. Shedding then terminates after an average of 11 days, at which point the acutely infectious cat becomes chronically infected. Recall that we assume no oocysts are shed during chronic infection. Once in the environment, T. gondii oocysts are very hardy, taking roughly a year to break down naturally in the absence of extenuating circumstances, such as extreme temperature [24]. Therefore, our model assumes T. gondii is shed into an ideal environment, such as warm and moist soil, with no interventions to remove oocysts [24]. Furthermore, loss of oocysts to cat infection is considered negligible as it only takes a few oocysts amongst the millions available to infect a cat. Using these numbers, we calculated the parameter values found in Table 1. For more insight on where the ranges come from, please see the Appendix.

Due to limited available data about the rate of infection, we use our model to test the effects of a range of values. This is best understood as two different parameters: the probability of infection when a susceptible cat encounters the parasite and the rate at which a cat encounters the parasite. Our model considers infection occurring by indirect transmission via contact with contaminated soil.

\section{Results}

The model (Equations (1) - (4)) is solved numerically using the $\mathrm{R}$ [21] programming language with the package deSolve [23]. Other symbolic computations are done using the program Mathematica [17].

\subsection{Rate of Infection}

Beginning with a total population of 500 cats containing a $1 \%$ acutely infectious cat subpopulation, Figure 2 illustrates the number of susceptible, acutely infectious, and chronically infectious cats with baseline parameter values from Table 1. Time is measured in weeks and the system was run for a length of 2600 weeks, or 50 years.

Observe the immediate surge of chronically infectious cats that occurs in Figures 2/A and $2 \mathrm{C}$. This is due to the environment becoming suddenly inundated with T. gondii from the acutely infectious cats, which rapidly transition to chronically infectious. Also, dynamics exhibit an initial narrow peak of acutely infectious cats; this is also likely due to the early influx of the parasite. Though the acutely infectious cats appear to vanish rapidly, the inset graphs show the transient dynamics on a shorter time scale. Acute infection lasts roughly eleven days, so the transition from the acutely infectious to the chronically infectious subpopulation is almost immediate on the timescale considered.

All populations display decaying oscillations and eventually level out at steady equilibrium values. As seen in Figure 2, each subpopulation tends to exhibit similar dynamics, but with different frequencies and amplitudes. Depending on the rate of infection, $\omega$, the equilibrium value of susceptible cats could be higher or lower than the equilibrium population of chronically infectious cats. In fact, there is a specific rate of infection at which the equilibria of these subpopulations are equal: the "critical rate of infection," as we call it. Below this value, the equilibrium number of chronically infectious cats will never outnumber 

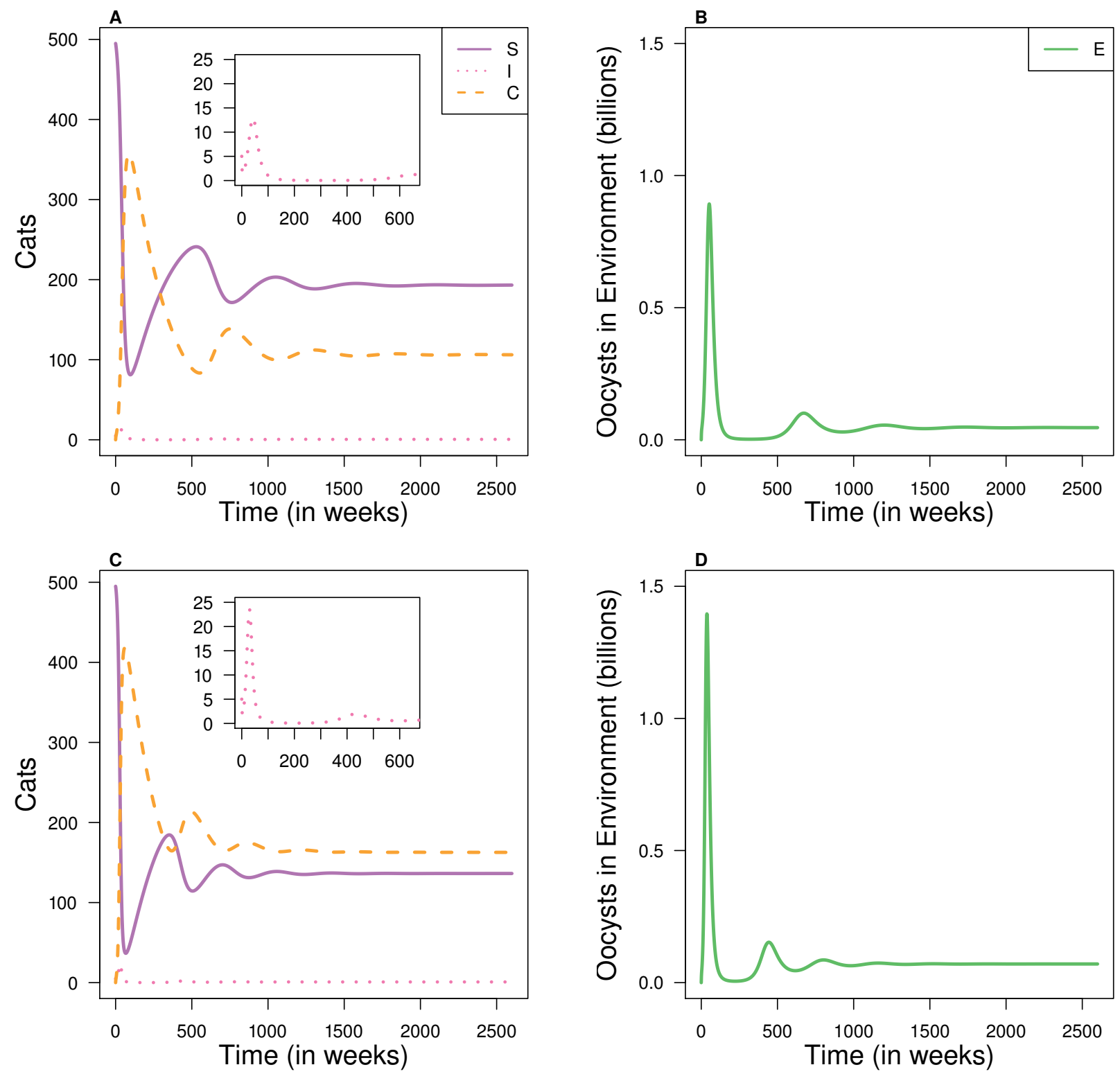

Figure 2: Dynamics with parameters $\omega \approx 0.046$ (in panels $\mathrm{A}$ and $\mathrm{B}$ ) and $\omega \approx 0.065$ (in panels $\mathrm{C}$ and $\mathrm{D}$ ). Inlaid are transient dynamics for acutely infectious cats on a shorter time scale. Baseline parameter values from Table 1 are used for all other parameters, with $\lambda=\frac{7}{11} \times 10^{7}$. Color and line styles are Susceptible $(S)$ : purple, solid; Acutely Infectious (I): pink, dotted; Chronically Infectious $(C)$ : orange, dashed; and Oocysts in Environment $(E)$ : green, solid. 
the susceptible cat subpopulation. Above this value, Toxoplasma gondii infection dominates. Calculations for this value for the different oocyst shedding rates follow in the next section. As we expand upon in the discussion, we define this as an indicator of the overall health of the population.

\subsection{Oocyst Shedding Rate}

Because transmission directly depends on how many oocysts are in the environment, the oocyst shedding rate $\left(\lambda_{I}\right)$ is also key to understanding the transmission dynamics. For Figure 2, we consider the baseline shedding rate of $10^{7}$ oocysts per cat per week during acute infection. Now we set the change in each subpopulation to zero (e.g., $\frac{d S}{d t}=0$ ) to find the equilibria. The expressions for each type of equilibrium are given in Table 2 , with further discussion below.

Table 2: Equilibrium values for $S^{*}, I^{*}, C^{*}$, and $E^{*}$.

\begin{tabular}{|ccc|}
\hline & Eradication & Endemic \\
\hline \hline$S^{*}$ & $\frac{b}{m}$ & $\frac{m \delta_{C} \eta\left(\gamma+\delta_{I} m\right)}{\omega\left(\gamma \lambda_{C}+\delta_{C} \lambda_{I} m\right)}$ \\
$I^{*}$ & 0 & $\frac{b \omega\left(\gamma \lambda_{C}+\delta_{C} \lambda_{I} m\right)-m^{2} \delta_{C} \eta\left(\gamma+\delta_{I} m\right)}{\omega\left(\gamma \lambda_{C}+\delta_{C} \lambda_{I} m\right)\left(\gamma+\delta_{I} m\right)}$ \\
$C^{*}$ & 0 & $\frac{\gamma\left(b \omega\left(\gamma \lambda_{C}+\delta_{C} \lambda_{I} m\right)-m^{2} \delta_{C} \eta\left(\gamma+\delta_{I} m\right)\right)}{m \delta_{C} \omega\left(\gamma \lambda_{C}+\delta_{C} \lambda_{I} m\right)\left(\gamma+\delta_{I} m\right)}$ \\
$E^{*}$ & 0 & $\frac{b \omega\left(\gamma \lambda_{C}+\delta_{C} \lambda_{I} m\right)-m^{2} \delta_{C} \eta\left(\gamma+\delta_{I} m\right)}{m \delta_{C} \eta \omega\left(\gamma+\delta_{I} m\right)}$ \\
\hline
\end{tabular}

For each shedding rate, Figure 3 displays the equilibrium values of each model variable as a function of rate of infection. We see a temporary plateau in equilibria before a more noticeable change with increasing $\omega$. During the plateau (at low $\omega$ values), susceptible cats are the only non-zero subpopulation. In this case, which we call the "eradication" or "infection-free" equilibrium, the parasite is eradicated and infection is no longer possible. Observe that the eradication equilibrium is stable until a certain rate of infection. As $\omega$ increases beyond this point, the eradication equilibrium becomes unstable and a new stable equilibrium emerges. We call this new type of equilibrium the "endemic equilibrium." That is, T. gondii will always be present in our cat population when the model exhibits this behavior. Notice that the switch from eradication to endemic equilibrium occurs at much smaller rates of infection when the shedding rate is on the order of $10^{8}$ compared to $10^{7}$ oocysts per cat per week. In subsection 3.2.1 we perform stability analysis to further study the stable endemic equilibrium. 

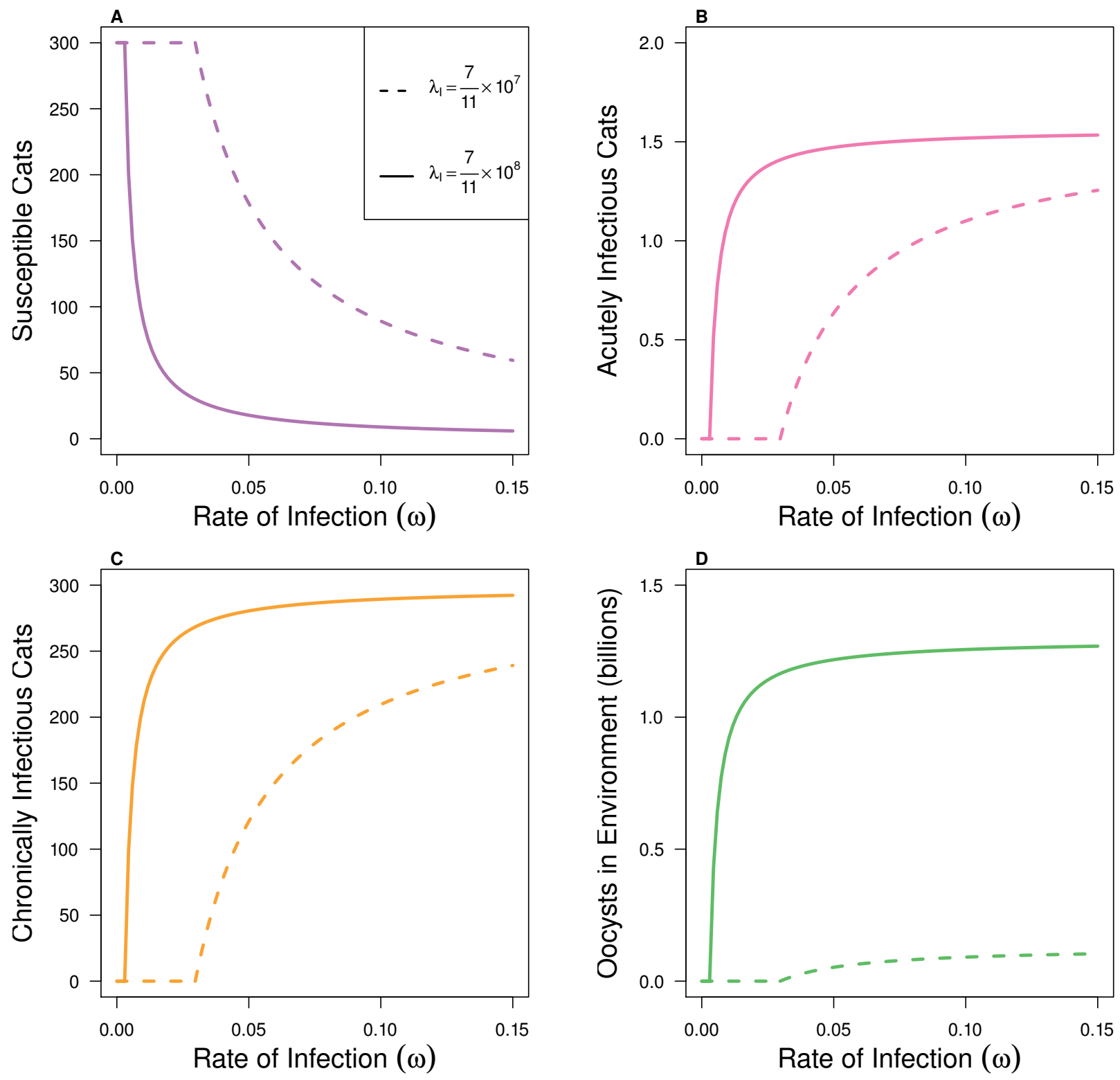

Figure 3: Equilibrium values for each variable (panel A: Susceptible $(S)$; panel B: Acutely Infectious (I); panel C: Chronically Infectious $(C)$; and panel D: Oocysts in Environment $(E)$ ), for two different oocyst shedding rates $\left(\lambda_{I}\right)$ across a range of rates of infection $(\omega)$. Baseline parameter values from Table 1 are used for all other parameters. 

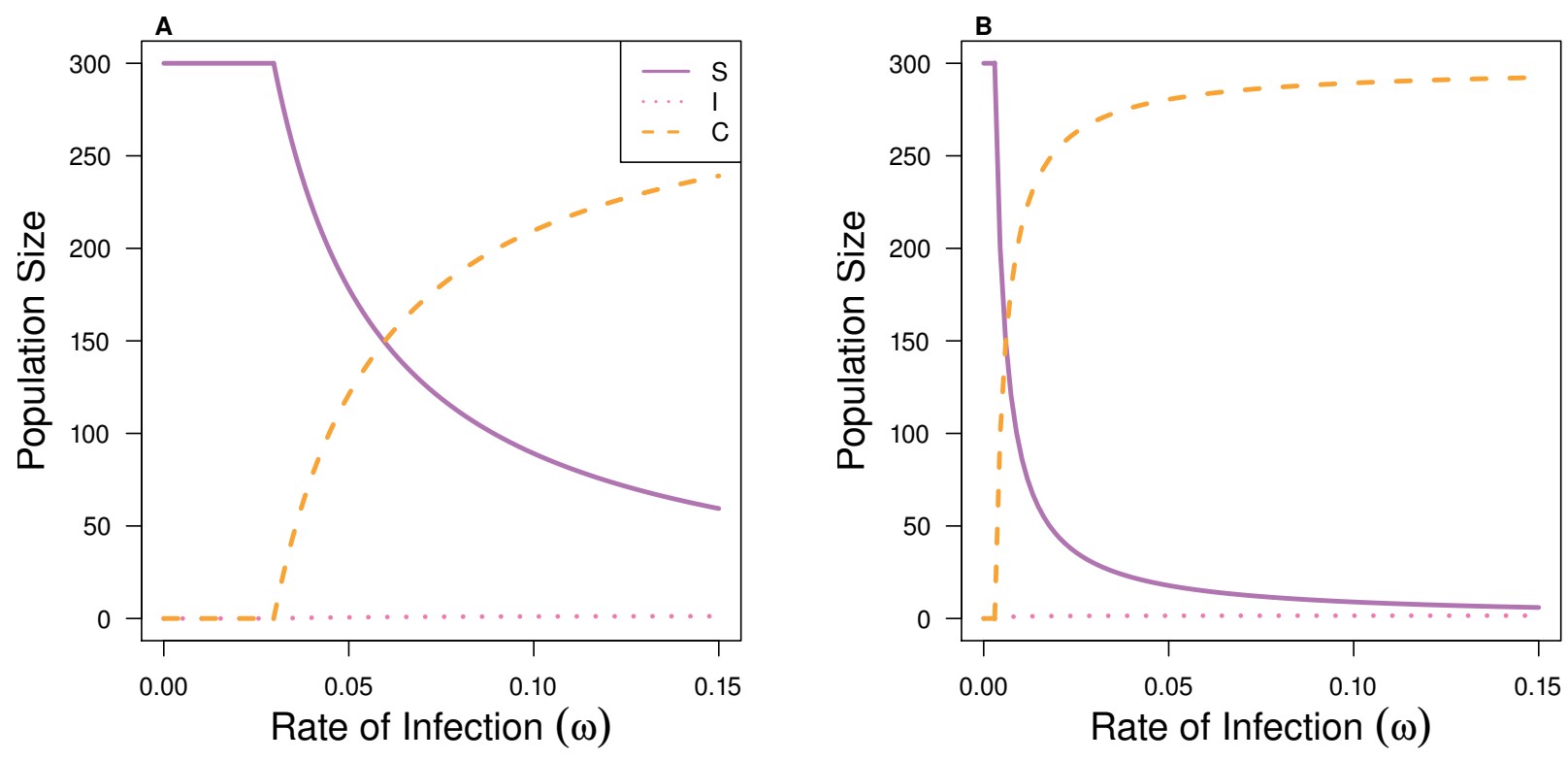

Figure 4: Equilibrium values for each cat subpopulation across a range of rates of infection $(\omega)$ for two different oocyst shedding rates $\left(\lambda_{I}\right)$. Baseline parameter values from Table 1 are used, with baseline shedding rate of $\lambda_{I}=\frac{7}{11} \times 10^{7}$ (in panel A) and maximum shedding rate of $\lambda_{I}=\frac{7}{11} \times 10^{8}$ (in panel B). Color and line styles are Susceptible (S): purple, solid; Acutely Infectious (I): pink, dotted; and Chronically Infectious $(C)$ : orange, dashed.

The effect of infection rate on cat equilibrium values is shown in Figure 4 . Note that Figure $4 \mathrm{~A}$ uses the same oocyst shedding rate as Figure 2. Also, recall the definition of the critical rate of infection: the rate of infection at which the equilibria for both the susceptible cats and chronically infectious cats are equal. To analytically find this value, we set endemic $S^{*}=C^{*}$ and solve for $\omega$. Thus, we define the critical rate of infection as

$$
\omega=m^{2} \eta \delta_{C}\left(\gamma+\delta_{I} m\right)\left(\frac{\delta_{C}\left(\gamma+\delta_{I} m\right)+\gamma}{\gamma b\left(\gamma \lambda_{C}+\delta_{C} \lambda_{I} m\right)}\right)
$$

Using baseline parameter values from Table 1, we find the critical infection rate to be $\omega \approx 0.0596$. This is consistent with the results in Figure 4A. At this point, the amount of oocysts in the environment is approximately 0.0649 billion. In Figure $4 \mathrm{~B}$, the critical value occurs at $\omega \approx 0.0059$. Unless the rate of infection is below this value, we see that chronically infectious cats will always dominate. This small value may not be attainable, and thus it will be difficult control T. gondii in a population shedding oocysts at a rate of $\lambda_{I}=\frac{7}{11} \times 10^{8}$. The amount of oocysts in the environment in this case is approximately 0.6492 billion.

\subsubsection{Stability Analysis}

Based on numerical and analytical results, there are two different types of equilibria: eradication and endemic. We calculate the basic reproductive number $\left(\mathcal{R}_{0}\right)$ to identify the stability of each equilibrium. Briefly, $\mathcal{R}_{0}$ is the expected number of secondary infections per primary 
infection [5]. Employing the method of the Next Generation Matrix (NGM) [6], we find the transmission matrix $(F)$ and transition matrix $(V)$ to be:

$$
F=\left[\begin{array}{ccc}
0 & 0 & \frac{b \omega}{m} \\
0 & 0 & 0 \\
0 & 0 & 0
\end{array}\right] \quad V=\left[\begin{array}{ccc}
\gamma+\delta_{I} m & 0 & 0 \\
-\gamma & \delta_{C} m & 0 \\
-\lambda_{I} & -\lambda_{C} & \eta
\end{array}\right]
$$

This gives our NGM:

$$
F V^{-1}=\left[\begin{array}{ccc}
\frac{b \omega\left(\gamma \lambda_{C}+\delta_{C} \lambda_{I} m\right)}{m^{2} \delta_{C} \eta\left(\gamma+\delta_{I} m\right)} & \frac{b \omega \lambda_{C}}{m^{2} \delta_{C} \eta} & \frac{b \omega}{m \eta} \\
0 & 0 & 0 \\
0 & 0 & 0
\end{array}\right]
$$

The dominant eigenvalue of $F V^{-1}$ is $\mathcal{R}_{0}$. For our model,

$$
\mathcal{R}_{0}=\frac{b \omega\left(\gamma \lambda_{C}+\delta_{C} \lambda_{I} m\right)}{m^{2} \delta_{C} \eta\left(\gamma+\delta_{I} m\right)}
$$

Recall, we focus on the rate of infection $(\omega)$ and oocyst shedding rate of acutely infectious cats $\left(\lambda_{I}\right)$ being the main drivers of $T$. gondii transmission in cats. For a fixed shedding rate, $R_{0}$ is linear over the rate of infection, and vice versa. When $\mathcal{R}_{0}<1$, the eradication equilibrium is stable and T. gondii is no longer an issue. We are concerned with the point at which eradication becomes unstable and the endemic equilibrium becomes stable. This occurs when $\mathcal{R}_{0}>1$. Using baseline parameter values from Table 1, we find the formula for the rate of infection at which endemic infection occurs,

$$
\omega>\frac{1.8907 \times 10^{-4}}{\lambda_{I}}
$$

This is $\omega>0.0297$ and $\omega>0.0029$ for the baseline and maximum shedding rate, respectively. The exchange in stability between eradication and endemic equilibrium is seen in Figure 3 .

Furthermore, notice that $\omega$ and $\lambda_{I}$ have an inverse relationship. As the oocyst shedding rate $\left(\lambda_{I}\right)$ decreases, the rate of infection $(\omega)$ must take on larger values in order for $T$. gondii to become endemic. Likewise, as the shedding rate increases, the rate of infection needed for a stable endemic equilibrium becomes fairly small. Ergo, as long as the rate of infection is small enough, eradication of T. gondii will occur.

\section{Discussion}

We built a differential equations model to explore the dynamics of a cat population exposed to Toxoplasma gondii. The model considered three cat subpopulations and the surrounding 
environment. We found two equilibria whose existence and stability was based on two key parameters: the oocyst shedding rate and rate of infection in cats. In transient and long-term dynamics, chronically infectious cats outnumber acutely infectious cats (Figure 2). Based on the rate of infection and shedding rate, long-term dynamics show either susceptible cats outnumbering chronically infectious cats, or vice versa. We define a healthy population to be one for which the susceptible subpopulation dominates. Though we have neglected chronic shedding, chronically infectious cats still harbor the parasite. For instance, a decaying stillinfected cat corpse can disperse the parasite into the soil and/or water or to scavengers. Furthermore, there have been cases in which T. gondii resurfaces after a dormant period during chronic infection [7, 8, 27]. These factors contribute to the success of the parasite, and future modeling work could investigate chronic shedding $\left(\lambda_{C} \neq 0\right)$.

For baseline parameters considered in the model, $\mathcal{R}_{0}<1$ if $\omega<\frac{1.8907 \times 10^{-4}}{\lambda_{I}}$. This corresponds to a stable eradication, or infection-free, equilibrium. More interesting dynamics and results occur for $\mathcal{R}_{0}>1$. We find that $\mathcal{R}_{0}>1$ for $\omega>0.0297$ with a baseline shedding rate, and $\omega>0.0029$ for a maximum shedding rate. Within this parameter regime, one strategy for enhancing the health of the population is to lower the rate of infection. By decreasing this rate, there are fewer infectious cats, hence fewer oocysts are shed into the environment, and a susceptible cat is not as likely to become infected. Alternatively, lowering the shedding rate of oocysts decreases the amount of the parasite in the environment, resulting in fewer new infections. So, once the rate of infection is under the critical value, the susceptible cats will dominate. Therefore, we propose to first aim for reducing the rate of infection under the critical value, with the ultimate goal of obtaining eradication.

Perhaps the development of a medication or treatment could reduce the oocyst shedding rate, but it is hard to imagine how to successfully medicate stray cats. A more viable option could be to treat the environment with an agent that kills T. gondii oocysts. This would lower the rate of infection by reducing the rate at which susceptible cats come in contact with the parasite. Hence, fewer cats become infected, resulting in less spillover to other species such as humans and the monk seal, effectively stopping T. gondii before it has a chance to pounce.

The current results are based on a model that considers an indirect form of transmission. Future models will incorporate a more explicit form by accounting for details such as consumption of infected prey. Including this direct transmission may elucidate other prevention techniques; for instance, reducing infection in prey might lower the rate of infection in cats. Furthermore, we will consider more concomitants of T. gondii, like intermittent chronically infectious shedding, as they may be a more authentic illustration of different cat population dynamics.

\section{Acknowledgments}

This project has benefitted from a supportive research environment for undergraduate students at the University of Central Oklahoma as well as research and travel grants from the Office of Research and Sponsored Programs, the Office of High-Impact Practices, the Research, Creative, and Scholarly Activities (RCSA) student-initiated grant program, and the College of Mathematics and Science CURE-STEM program. 


\section{References}

[1] Pierre Ambroise-Thomas and Eskild Petersen. Congenital Toxoplasmosis: Scientific Background, Clinical Management and Control. Springer Science \& Business Media, 2013.

[2] Mariza M Avelino, Waldemar N Amaral, Isolina MX Rodrigues, Alan R Rassi, Maria BF Gomes, Tatiane L Costa, and Ana M Castro. Congenital toxoplasmosis and prenatal care state programs. BMC Infectious Diseases, 14(1):33, 2014.

[3] Vern B Carruthers and Yasuhiro Suzuki. Effects of Toxoplasma gondii infection on the brain. Schizophrenia Bulletin, 33(3):745-751, 2007.

[4] Haydee A Dabritz, Melissa A Miller, E Robert Atwill, Ian A Gardner, Christian M Leutenegger, Ann C Melli, and Patricia A Conrad. Detection of Toxoplasma gondii-like oocysts in cat feces and estimates of the environmental oocyst burden. Journal of the American Veterinary Medical Association, 231(11):1676-1684, 2007.

[5] O. Diekmann, J. A. P. Heesterbeek, and J. A. J. Metz. On the definition and the computation of the basic reproduction ratio $\mathcal{R}_{0}$ in models for infectious diseases in heterogeneous populations. Journal of Mathematical Biology, 28(4):365-382, Jun 1990.

[6] O. Diekmann, J. A. P. Heesterbeek, and M. G. Roberts. The construction of nextgeneration matrices for compartmental epidemic models. Journal of The Royal Society Interface, $7(47): 873-885,2010$.

[7] JP Dubey. Duration of immunity to shedding of Toxoplasma gondii oocysts by cats. The Journal of Parasitology, pages 410-415, 1995.

[8] JP Dubey. Unexpected oocyst shedding by cats fed Toxoplasma gondii tachyzoites: in vivo stage conversion and strain variation. Veterinary Parasitology, 133(4):289-298, 2005.

[9] Zhilan Feng, Jorge Velasco-Hernandez, and Brenda Tapia-Santos. A mathematical model for coupling within-host and between-host dynamics in an environmentally-driven infectious disease. Mathematical Biosciences, 241(1):49-55, 2013.

[10] NOAA Fisheries. Hawaiian monk seal (Neomonachus schauinslandi). NOAA Fisheries, $6(25): 2012,2011$.

[11] Jaroslav Flegr, Joseph Prandota, Michaela Sovičková, and Zafar H Israili. Toxoplasmosis-a global threat. PloS one, 9(3):e90203, 2014.

[12] Centers for Disease Control \& Prevention. Parasites - Toxoplasmosis (Toxoplasma Infection). Centers for Disease Control and Prevention, 2016. Accessed: 2016-06-27.

[13] American Society for the Prevention of Cruelty to Animals. A Closer Look at Community Cats. ASPCA, 2017. Accessed: 2017-09-29. 
[14] JK Frenkel and JP Dubey. Toxoplasmosis and its prevention in cats and man. Journal of Infectious Diseases, 126(6):664-673, 1972.

[15] Gilberto C González-Parra, Abraham J Arenas, Diego F Aranda, Rafael J Villanueva, and Lucas Jódar. Dynamics of a model of toxoplasmosis disease in human and cat populations. Computers $\mathcal{E} 3$ Mathematics with Applications, 57(10):1692-1700, 2009.

[16] Shelley P Honnold, Robert Braun, Dana P Scott, C Sreekumar, and JP Dubey. Toxoplasmosis in a Hawaiian Monk Seal (Monachus schauinslandi). Journal of Parasitology, 91(3):695-697, 2005.

[17] Wolfram Research, Inc. Mathematica, Version 11.3. Champaign, IL, 2018.

[18] Jeffrey L Jones, Deanna Kruszon-Moran, Marianna Wilson, Geraldine McQuillan, Thomas Navin, and James B McAuley. Toxoplasma gondii infection in the United States: seroprevalence and risk factors. American Journal of Epidemiology, 154(4):357$365,2001$.

[19] Emily L Lilly and Caroline D Wortham. High prevalence of Toxoplasma gondii oocyst shedding in stray and pet cats (Felis catus) in Virginia, United States. Parasites 86 Vectors, 6(1):266, 2013.

[20] Felicia B Nutter, Jay F Levine, and Michael K Stoskopf. Reproductive capacity of freeroaming domestic cats and kitten survival rate. Journal of the American Veterinary Medical Association, 225(9):1399-1402, 2004.

[21] R Core Team. R: A Language and Environment for Statistical Computing. R Foundation for Statistical Computing, Vienna, Austria, 2017.

[22] National Marine Fisheries Service. Hawaiian Monk Seals and Toxoplasma gondii. National Oceanic and Atmospheric Association, 2016.

[23] Karline Soetaert, Thomas Petzoldt, and R. Woodrow Setzer. Solving differential equations in R: Package deSolve. Journal of Statistical Software, 33(9):1-25, 2010.

[24] Anna Rovid Spickler. Toxoplasmosis: Technical factsheet. Iowa State University, 2017.

[25] Mayo Clinic Staff. Diseases and Conditions: Toxoplasmosis. Mayo Clinic, 2016. Accessed: 2016-06-27.

[26] Paul R Torgerson and Pierpaolo Mastroiacovo. The global burden of congenital toxoplasmosis: a systematic review. Bulletin of the World Health Organization, 91(7):501-508, 2013.

[27] Dauton Luiz Zulpo, Ana Sue Sammi, Joeleni Rosa dos Santos, João Pedro Sasse, Thais Agostinho Martins, Ana Flávia Minutti, Sérgio Tosi Cardim, Luiz Daniel de Barros, Italmar Teodorico Navarro, and João Luis Garcia. Toxoplasma gondii: A study of oocyst re-shedding in domestic cats. Veterinary Parasitology, 249:17-20, 2018. 


\section{A Parameter Value Calculations}

The following are calculations for the range of values for the parameters found in Table 1. Midpoints of each parameter range were used to solve our model unless stated otherwise.

Birth rate $(b)$ : For this value, we want a constant number of cats born per week assuming an average of 30 litters per population of cats a year with an equal birth-sex ratio.

$$
\begin{aligned}
& \min \frac{30 \text { litters }}{1 \text { year }} \times \frac{2 \text { cats }}{1 \text { litter }} \times \frac{1 \text { female cat }}{2 \text { cats }} \times \frac{1 \text { year }}{52 \text { weeks }}=\frac{30 \text { female cats }}{52 \text { weeks }} \\
& \max \frac{30 \text { litters }}{1 \text { year }} \times \frac{6 \text { cats }}{1 \text { litter }} \times \frac{1 \text { female cat }}{2 \text { cats }} \times \frac{1 \text { year }}{52 \text { weeks }}=\frac{90 \text { female cats }}{52 \text { weeks }}
\end{aligned}
$$

Mortality rate $(\mathrm{m})$ : This parameter is also a constant number assuming a range of lifespans for feral, rather than domestic, cats.

$$
\begin{aligned}
& \min \frac{1 \text { cat }}{10 \text { years }} \times \frac{1 \text { year }}{52 \text { weeks }}=\frac{1 \text { cats }}{520 \text { weeks }} \\
& \max \quad \frac{3 \text { cats }}{10 \text { years }} \times \frac{1 \text { year }}{52 \text { weeks }}=\frac{3 \text { cats }}{520 \text { weeks }}
\end{aligned}
$$

Loss of acute infection $(\gamma)$ : Recall T. gondii oocysts are shed for 7-15 days during acute infection. This value reflects the length of the shedding period. Following this, the cat becomes chronically infectious.

$$
\begin{aligned}
& \min \frac{1}{15 \text { days }} \times \frac{7 \text { days }}{1 \text { week }}=\frac{7}{15 \text { weeks }} \\
& \max \\
& \frac{1}{7 \text { days }} \times \frac{7 \text { days }}{1 \text { week }}=\frac{1}{1 \text { week }}
\end{aligned}
$$

Infection associated mortality $\left(\delta_{j}\right)$ : Because of $T$. gondii's tendency to not harm its definitive host, we assume this value is only a slight increase beyond the natural mortality of a cat. Therefore, we take $\delta_{j}$ to be in the range of 1.005 to 1.05 . 
Oocyst breakdown in environment $(\eta)$ : We assume these minimum and maximum rates based on available literature.

$$
\begin{aligned}
& \min \frac{1}{\frac{1}{3} \text { year }} \times \frac{1 \text { year }}{52 \text { weeks }}=\frac{3}{52 \text { weeks }} \\
& \max \quad \frac{1}{\frac{1}{5} \text { year }} \times \frac{1 \text { year }}{52 \text { weeks }}=\frac{5}{52 \text { weeks }}
\end{aligned}
$$

Oocyst shedding rate $\left(\lambda_{j}\right)$ : Recall a range of $10^{6}-10^{8}$ oocysts are shed during acute infection per cat for an average of 11 days. These values reflect this.

$$
\begin{aligned}
& \min \frac{1}{1 \text { cat }} \times \frac{10^{6} \text { oocysts }}{11 \text { days }} \times \frac{7 \text { days }}{1 \text { week }}=\frac{7 \times 10^{6} \text { oocysts }}{11 \text { cat } \cdot \text { week }} \\
& \max \frac{1}{1 \text { cat }} \times \frac{10^{8} \text { oocysts }}{11 \text { days }} \times \frac{7 \text { days }}{1 \text { week }}=\frac{7 \times 10^{8} \text { oocysts }}{11 \text { cat } \cdot \text { week }}
\end{aligned}
$$

Rate of infection $(\omega)$ : Based on preliminary numerical investigations using well-documented parameters, this range, 0.01 to 0.15 , was chosen because it displays a broad range of dynamics including eradication of infection. 